\title{
A Conceptual Framework for Mental Model-based Repeated Multifaceted Intervention Design to Improve Preventive Health Behaviors and Outcomes
}

Mazbahul G Ahamad ( $\square$ mahamad@huskers.unl.edu )

University of Nebraska-Lincoln https://orcid.org/0000-0002-8765-1898

\section{Fahian Tanin}

Independent Researcher https://orcid.org/0000-0002-0872-8122

\section{Method Article}

Keywords: Public Health Intervention, Health Education and Promotion, Behavior Change Intervention, Intervention Design, Multifaceted Intervention, Repeated Intervention, Mental Model Mapping, Low- and Medium-Income Country (LMIC)

Posted Date: July 14th, 2020

DOl: https://doi.org/10.21203/rs.3.rs-41156/v1

License: (c) (i) This work is licensed under a Creative Commons Attribution 4.0 International License. Read Full License 


\section{Abstract}

Background: Improving the effectiveness of health interventions is a major challenge in public health research and program development. A large body of literature found low or no impact of health education and promotional interventions, especially in low- and middle-income countries.

Objectives: To develop a conceptual framework in support of intervention designs of preventive health behavior improvement programs and outcomes.

Methods: An overview of systematic and scoping reviews along with empirical literature was conducted to assess the limitations of preventive health education and promotion interventions using water, sanitation, and hygiene (WASH) programs and outcomes as examples.

Results: We analyzed the limitations of the less effective or ineffective field interventions cited in peerreviewed articles. We found three major limitations regarding the mental model's balance of treatment and comparison groups, treatment groups' willingness to adopt suggested behaviors, and the type, length, frequency, intensity, and sequence of treatments. To minimize the influence of these concerns, we proposed a mental model-based repeated multifaceted (MRM) intervention design framework.

Conclusions: Our proposed framework aims to provide an intervention design for improving health education and promotional programs. Further studies using this framework are essential to examine our hypotheses and advance the understanding of how MRM design can improve preventive health behaviors and outcomes.

\section{Background}

Increasing the effectiveness of interventions focused on improving preventive health behavior is a the major challenge in public health research and program development (1) due to the complex nature and context dependency of these behaviors (2-4). Intervention programs typically require a change in behavior among the members of a treatment group (e.g., individual, household) to improve preventive health behaviors and health outcomes (5). Behavior change interventions may be minimally effective or ineffective due to inappropriate experiment and treatment design, the influence of contextual factors (3), or unobserved events, especially in low- and middle-income countries (6-9).

In interventions focused on preventive health behavior, we have noticed three distinct concerns regarding low or no treatment and intervention effects. First, intervention approaches implicitly assume that all treated individuals or households are identical and have similar mental models or cognitive capacities; this increases the likelihood of sample imbalance and bias in outcome estimates (10). Sample balance tests frequently disregard behavioral or cognitive factors (11) but do consider observable socioeconomic and demographic characteristics (e.g., income, wealth) and, sometimes, knowledge levels regarding health behaviors. Second, behavior change interventions require a long time period (12), and treatment effects wane over time after single-treatment-based interventions (13). Third, the treatment groups' 
exposure to the intervention does not vary or repeat throughout the study period (14). These three elements can, in combination, be a significant intervention-related source of poor health behaviors and outcomes. A framework is needed to shape preventive health behavior intervention designs to consider concerns relating to balance tests, treatment effect waning, and impact persistency.

In this paper, we develop a conceptual framework for a mental model-based repeated multifaceted (MRM) intervention design to improve the effectiveness of preventive health behavior interventions. This approach suggests using the insights from mental model mapping of treatment groups. It incorporates the conviction that repeated and mixed interventions are necessary to generate persistent treatment effects, as preventive health behaviors are difficult to change. In addition, mixed treatment can be more effective and persistent than either financial or behavioral treatment alone.

\section{Methods}

\subsection{Literature Search and Identification of Key Concerns}

We conducted an overview of the literature, including systematic and scoping reviews and empirical articles, on the limitations of water, sanitation, and hygiene (WASH)-related health education and promotion interventions and outcomes, especially in low- and middle-income countries. We applied our search strategy to PubMed and Cochrane Library to source peer-reviewed articles published from January 2010 to June 2020 that used experimental field data (Table A1). Ultimately, 86 systematic reviews and 49 empirical articles from PubMed and 129 trials from Cochrane Library were identified as useful in exploring the limitations of existing design approaches and intervention outcomes.

\subsection{Framework Development}

Our proposed MRM intervention design framework is based on the major concerns cited in existing systematic and scoping reviews, trials, and recent empirical studies regarding approaches, frequency, treatment components, sample balance variables, and time dimensions of treatment interventions in published WASH-related field experiments (Table A2). We considered different experimental approaches used in other fields (e.g., natural resources, development economics) to develop a modified intervention design that minimizes limitations (Fig. 1).

\section{Results}

\subsection{Major Challenges in Program Interventions}

Based on our review of the literature, we found three major concerns in intervention design and implementation; these concerns underpin the theoretical foundation of our proposed framework.

First, standard intervention design assumes that treatment subjects are similarly willing to adopt suggested preventive behaviors and have similar cognitive capacities; in fact, individuals have different 
cognitive capacities and mental models (0). These differences might lead to low-level outcomes. Human behavior is influenced by human attitudes, knowledge, perceptions, social norms, and beliefs $(15,16)$; these elements together help constitute individuals' mental models. Therefore, it is crucial to identify whether one of these elements would affect intervention outcomes.

Second, the impact of single and short-term interventions wanes over time $(5,13)$, while lasting behavioral change requires long-term intervention (0).

Third, treatment groups' responses to a specific intervention can vary under different or changing circumstances; most of the time, this concern was not fully recognized. For example, a few individuals may prefer monetary incentives to behavioral nudging while unemployed. In addition, some treatment groups may respond better to behavioral nudging and hands-on experience than other groups (0).

\subsection{Conceptual Framework}

The MRM intervention design framework takes mental model mapping to be essential and further includes two core ideas: multifaceted intervention and repeated interventions (see Fig. 1).

First, mapping mental models in the design stage allows the researcher or program analyst to understand a treatment group's mental or cognitive ability to adopt suggested preventive behaviors as well as their willingness to do so (Stage 1 of Fig. 1). Sample balance tests need to consider mental model-related variables (e.g., flexibility) together with socioeconomic and demographic variables, such as age, sex, education, income, and wealth. Mapping mental models before and after each intervention is particularly important so that any changes can be identified across time and treatments.

Second, multifaceted intervention allows multiple-component treatments and can generate more lasting effects than single-component interventions (17). For example, different types of educational, financial, and behavioral interventions with different intensity levels (e.g., low, standard, and high) can be combined to design a treatment package (Stage 2 of Fig. 1). However, the length and intensity of the treatment may differ and should be tailored to relevant behavior-related outcomes (Table 1). In some cases, both monetary and behavioral interventions are essential to encourage the treatment groups to adopt behavior.

Third, repeated interventions may produce more pronounced intervention outcomes (Stage 2 of Fig. 1) and are more effective than single intervention. While the impact of a single intervention wanes over time, mixed interventions in multiple rounds are more likely to produce anticipated outcomes.

\section{Discussion}

Due to the low efficacy of interventions for preventive health behaviors, there is a need for alternative designs that can produce better health outcomes. The MRM framework proposes a basic intervention 
design with three main features: mental model mapping, repeated interventions, and multifaceted interventions.

\subsection{Design and Implementation}

In Stage 1, mapping the mental models of a treatment group before a baseline survey is essential to designing the initial intervention (Fig. 1). A mental model is an overall representation of an individual's characteristics (e.g., attitudes, values, beliefs, social and cultural norms) that explains that individual's reasoning, inferring, and decision-making processes. These processes influence an individual's ability to grasp and willingness to accept suggested health behaviors (18-22). Mapping mental models using modified versions of available methods $(19,22,23)$ would provide insights into an individual's or household's behavioral and cognitive capacity as they relate to the adoption of suggested health behaviors.

Table 1: An Example of Mental Model-based Repeated Multifaceted (MRM) Intervention Design.

\begin{tabular}{|c|c|c|c|c|c|c|}
\hline \multirow[t]{2}{*}{ Round } & \multicolumn{6}{|c|}{ Intervention Component } \\
\hline & Feature & Comp. \#1 & Comp. \#2 & Comp. \#3 & Comp. \#4 & Comp. \#5 \\
\hline \multirow[t]{4}{*}{ Initial } & Type & Informational & & & & \\
\hline & Length & Short-term & & & & \\
\hline & Intensity & Standard & & & & \\
\hline & Evaluate & with baseline & & & & \\
\hline \multirow[t]{4}{*}{ Int. \#1 } & Type & Practical & Behavioral & & & \\
\hline & Length & Short-term & Medium-term & & & \\
\hline & Intensity & Standard & Low-level & & & \\
\hline & Evaluate & with baseline & nd initial interv & ntions & & \\
\hline \multirow[t]{4}{*}{ Int. \#2 } & Type & Informational & Practical & Financial & Promotional & \\
\hline & Length & Short-term & Medium-term & Short-term & Short-term & \\
\hline & Intensity & Minimum & Low-level & Standard & High-level & \\
\hline & Evaluate & with baseline, & nitial, and inter & nediate \# 1 i & erventions & \\
\hline \multirow[t]{4}{*}{ Final } & Type & Informational & Practical & Behavioral & Financial & Promotional \\
\hline & Length & Short-term & Medium-term & Short-term & Short-term & Short-term \\
\hline & Intensity & Low-level & High-level & Low-level & Standard & Low-level \\
\hline & Evaluate & \multicolumn{5}{|c|}{ with baseline, initial, intermediate \#1, and intermediate \#2 interventions } \\
\hline
\end{tabular}

Notes: Int. = Intermediate; Comp. = Component.

Stage 2 includes two different types of intervention: initial and intermediate (Fig. 1). The number of intermediate interventions as well as their type and sequence (e.g., informational-practical-behavioral, informational-behavioral-practical) should be adjusted in line with a program's short- and long-term goals. Researchers will need to identify the appropriate length (e.g., short, medium, long) and intensity (e.g., low, standard, high) of each treatment, depending on the research goals.

In the final evaluation (Stage 3), researchers compare the final outcomes with the baseline and intermediate outcomes to arrive at conclusions regarding specific stage-level outcomes. Redesign is necessary if the initial treatment produced lower-than-expected outcomes. 
A hypothetical MRM intervention design is presented in Table 1 with further elaboration. Each component has three distinct features in terms of intervention type (e.g., informational, educational, financial, behavioral), length (e.g., short-, medium-, long-term), and intensity (e.g., low, standard, high). A standard information component can be employed in the short-term in the initial stage. In the final stage, five different components can be employed sequentially as a treatment package. This sequence could be a cluster of mixed interventions where the order of interventions (and their close variants) are based on the mental models of the treatment group members and expected outcomes from the programs. Thus, individuals with limited learning or adoption capacity, for instance, could be treated with higher intensity.

\subsection{Outcome Analysis}

As each intervention combines multiple treatments, researchers should consider all the treatments at a given stage as a treatment package (e.g., treatments 1,2 , and 3 are a treatment package for intermediate intervention \#2). Comparing the outcomes of each intervention with previous interventions (e.g., comparing intermediate intervention \#2 with the baseline and with intermediate intervention \#1) is required to reveal whether the effects of treatment packages persist; this information can be used to shape long-term public health policy. The possibility of courtesy bias (6) by the responders during afterintervention data collection should be accounted for when promotional components are included as part of a treatment package.

Most notably, researchers need to check to the mental model after each intervention to compare the initial mental model, the subject's willingness to accept the behavioral change (as stated in a baseline survey), and the subject's actual or demonstrated willingness to accept the suggested behaviors. Sub-group analysis is essential to assess adherence to suggested behaviors by different groups within or between treatment groups. A crossover design allows for various evaluation techniques, such as quasiexperimental designs (e.g., pre-post) for initial interventions and experimental designs (e.g., difference-indifference) for intermediate and final interventions.

\subsection{Implementation Challenges}

Intervention programs designed according to the proposed framework will incur some challenges in the design, field application, and evaluation stages.

First, individual or household-level mental models vary contextually; therefore, a suitable mental model mapping technique needs to be adopted. Classification of these models requires careful understanding of experimental contexts. A professional behavioral profiler is needed to ensure accuracy, as typical enumerators are not trained to perform mental model mapping.

Second, identification of the appropriate length and intensities of different treatments would be challenging during the initial and first intermediate stages due to various contextual factors. Researchers can use these two stages to test initial treatments and identify appropriate treatment conditions to employ in the later stages. 
Third, the effect size of intermediate interventions may be misleading due to a variety of outside factors (e.g., unexpected bad or good weather). The average treatment effect, in this case, could be used as a proxy indicator of effect size. Overall, this approach requires adequate financial and human resources to employ multiple interventions, subsequent assessments, and treatment redesign.

\subsection{Public Health Implications}

This conceptual framework for MRM intervention design offers an alternative intervention approach to improve individual and household-level preventive health behaviors and outcomes by enabling more comprehensive treatment designs for interventions. This approach can be applied to any behavioral change interventions. Additional field experiments using this framework should be employed to assess whether the proposed MRM intervention design improves health behaviors and outcomes.

\section{Declarations}

Draft Version: July 10, 2020.

Authors' Contributions: MGA conceived the original idea and developed the logical sequence of the intervention design flowchart, interpreted data from the overview of published articles, and wrote the first draft using literature jointly sourced by MGA and FT. MGA and FT are now revising the draft.

Ethical approval: Ethical approval was not sought, as we used secondary meta-data on the limitations of program interventions cited in peer-reviewed articles and working papers.

Competing interest: None declared.

Funding: None.

\section{References}

1. Dreibelbis R, Winch $P$, Leontsini E, Hulland K, Ram P, Unicomb L, et al. The integrated behavioural model for water, sanitation, and hygiene: A systematic review of behavioural models and a framework for designing and evaluating behaviour change interventions in infrastructure-restricted settings. BMC Public Health. 2013 Dec 26;13:1015:2-13.

2. Rychetnik L, Frommer M, Hawe P, Shiell A. Criteria for evaluating evidence on public health interventions. J Epidemiol Community Health. 2002 Feb 1;56(2):119-27.

3. Fewtrell L, Kaufmann R, Kay D, Enanoria W, Haller L, Colford J. Water, sanitation, and hygiene interventions to reduce diarrhoea in less developed countries: A systematic review and metaanalysis. Lancet Infect Dis. 2005 Jan 1;5(1):42-52.

4. Hawe P. Lessons from complex interventions to improve health. Annu Rev Public Health. 2015 Mar 18;36(1):307-23. 
5. White H, Raitzer D. Impact Evaluation of Development Interventions: A Practical Guide. Manila: Asian Development Bank; 2017. 1-217 p.

6. Luby S, Rahman M, Arnold B, Unicomb L, Ashraf S, Winch P, et al. Effects of water quality, sanitation, handwashing, and nutritional interventions on diarrhoea and child growth in rural Bangladesh: a cluster randomised controlled trial. Lancet Glob Heal. 2018 Mar 1;6(3):e302-15.

7. Null C, Stewart C, Pickering A, Dentz H, Arnold B, Arnold C, et al. Effects of water quality, sanitation, handwashing, and nutritional interventions on diarrhoea and child growth in rural Kenya: a clusterrandomised controlled trial. Lancet Glob Heal. 2018 Mar 1;6(3):e316-29.

8. Humphrey J, Mbuya M, Ntozini R, Moulton L, Stoltzfus R, Prendergast A, et al. Independent and combined effects of improved water, sanitation, and hygiene, and improved complementary feeding, on child stunting and anaemia in rural Zimbabwe: a cluster-randomised trial. Lancet Glob Heal. 2019;7(1):132-79.

9. Gera T, Shah D, Sachdev H. Impact of water, sanitation and hygiene interventions on growth, nondiarrheal morbidity and mortality in children residing in low- and middle-income countries: A systematic review. Indian Pediatr. 2018 May 1;55(5):381-93.

10. Buchmann N, Field E, Glennerster R, Hussam R. Throwing the baby out with the drinking water: Unintended consequences of arsenic mitigation efforts in Bangladesh. Cambridge, MA; 2019 Apr.

11. Mosler H-J. A systematic approach to behavior change interventions for the water and sanitation sector in developing countries: a conceptual model, a review, and a guideline. Int J Environ Health Res. 2012 Oct 1;22(5):431-49.

12. Michie S, West R, Sheals $K$, Godinho $C$. Evaluating the effectiveness of behavior change techniques in health-related behavior: a scoping review of methods used. Transl Behav Med. 2018;8(2):212-24.

13. Ferraro $P$, Price $M$. Using nonpecuniary strategies to influence behavior: Eevidence from a large-scale field experiment. Rev Econ Stat. 2013 Mar 19;95(1):64-73.

14. Craig P, Katikireddi S, Leyland A, Popham F. Natural experiments: An overview of methods, approaches, and contributions to public health intervention research. Annu Rev Public Health. 2017 Mar 20;38(1):39-56.

15. Michie S, van Stralen M, West R. The behaviour change wheel: A new method for characterising and designing behaviour change interventions. Implement Sci. 2011 Apr 23;6(1):42.

16. Thaler RH. Mental accounting matters. J Behav Decis Mak [Internet]. 1999 Sep 1 [cited 2020 Jul 1];12(3):183-206. Available from:

https://onlinelibrary.wiley.com/doi/full/10.1002/\%28SICI\%291099-

0771\%28199909\%2912\%3A3\%3C183\%3A\%3AAID-BDM318\%3E3.0.CO\%3B2-F

17. Banerjee A, Duflo E, Goldberg N, Karlan D, Osei R, Pariente W, et al. A multifaceted program causes lasting progress for the very poor: Evidence from six countries. Science (80- ). 2015 May 15;348(6236):1260799-1260799.

18. Ahamad MG. Mental model mapping for behavioral program design. Behavioural and Social Sciences at Nature Research [Internet]. 2019 [cited 2020 Jul 1]; Available from: 
https://socialsciences.nature.com/posts/48694-mental-model-mapping-for-behavioral-design

19. Datta S, Mullainathan S. Behavioral design: A new approach to development policy. Rev Income Wealth. 2014 Mar 1;60(1):7-35.

20. Ajzen I. The theory of planned behavior. Organ Behav Hum Decis Process. 1991 Dec 1;50(2):179211.

21. World Bank. Thinking with mental models. In: World Bank Group, editor. World Development Report 2015: Mind, Society, and Behavior. Washington, DC; 2015. p. 62-77.

22. Kelly M, Barker M. Why is changing health-related behaviour so difficult? Public Health. 2016 Jul 1;136:109-16.

23. Hoffman $M$, Lubell $M$, Hillis V. Linking knowledge and action through mental models of sustainable agriculture. Proc Natl Acad Sci. 2014 Sep 9;111(36):13016-21.

24. López-Mosquera N, Sánchez M. Theory of planned behavior and the value-belief-norm theory explaining willingness to pay for a suburban park. J Environ Manage. 2012 Dec 30;113:251-62.

\section{Appendix}

Table A1: Literature Search Strategy for MRM Intervention Design, 2010-2020.

\begin{tabular}{|l|l|l|}
\hline Database & Query & Results \\
\hline PubMed & $\begin{array}{l}\text { Search: (((water) AND (sanitation)) AND (hygiene)) AND (WASH) } \\
\text { Filters: Meta-Analysis, Review, Systematic Review, in the last 10 years }\end{array}$ & 80 \\
\cline { 2 - 3 } & $\begin{array}{l}\text { Search: (((water) AND (sanitation)) AND (hygiene)) AND (WASH)) AND (behavioral } \\
\text { change) } \\
\text { Filters: Systematic Review, in the last 10 years }\end{array}$ & 6 \\
\cline { 2 - 3 } & $\begin{array}{l}\text { Search: (((water) AND (sanitation)) AND (hygiene)) AND } \\
\text { (WASH)) Filters: Randomized Controlled Trial, in the last 10 years }\end{array}$ & 49 \\
\hline $\begin{array}{l}\text { Cochrane } \\
\text { Library }\end{array}$ & $\begin{array}{l}\text { (water):ti,ab,kw AND (sanitation):ti,ab,kw AND (hygiene):ti,ab,kw AND } \\
\text { (WASH):ti,ab,kw (Word variations have been searched) " with Publication Year from } \\
\text { 2010 to 2020, in Trials (Word variations have been searched) }\end{array}$ & 129 \\
\hline
\end{tabular}

Table A2: Major Features and Concerns of Preventive Health Behavior-related Interventions. 


\begin{tabular}{|l|l|l|l|}
\hline Issues & Category & Key Feature & Concern/Advantage \\
\hline \multirow{4}{*}{ Approach } & Single & Only one treatment & Treatment effect wanes over time \\
\cline { 2 - 4 } & Multifaceted & Multiple treatment & Persistent treatment effect \\
\hline Brequency & Single intervention & One round & Treatment effect wanes over time \\
\cline { 2 - 4 } test & Multiple intervention & Several round & Creates more persistent effect \\
\hline \multirow{3}{*}{$\begin{array}{l}\text { Treatment } \\
\text { component }\end{array}$} & $\begin{array}{l}\text { Socioeconomic and } \\
\text { demographic factors }\end{array}$ & $\begin{array}{l}\text { Income, wealth, age, } \\
\text { sex, education }\end{array}$ & $\begin{array}{l}\text { Mental model or cognitive capacity-related } \\
\text { factors are mostly absent }\end{array}$ \\
\cline { 2 - 4 } & Educational & $\begin{array}{l}\text { Information-based } \\
\text { letter }\end{array}$ & Less effective \\
\cline { 2 - 4 } & Training & Education & Effective but depends on the curriculum \\
\cline { 2 - 4 } & Financial & Hands-on experience & $\begin{array}{l}\text { Effective but depends on the type and } \\
\text { length of training }\end{array}$ \\
\cline { 2 - 4 } & Promotional & In cash only & Attractive but ineffective if stopped \\
\cline { 2 - 4 } & Behavioral & In kind or service & High effective with other treatments \\
\cline { 2 - 4 } & Mixed & $\begin{array}{l}\text { Weak or strong norm- } \\
\text { based nudging }\end{array}$ & $\begin{array}{l}\text { High effective with other financial } \\
\text { treatments }\end{array}$ \\
\cline { 2 - 4 } & & $\begin{array}{l}\text { Both financial and } \\
\text { behavioral }\end{array}$ & $\begin{array}{l}\text { Effective than either financial or behavioral } \\
\text { alone }\end{array}$ \\
\hline \multirow{4}{*}{$\begin{array}{l}\text { Time } \\
\text { dimension }\end{array}$} & Short-term & Less than one year & Treatment effects wanes over time \\
\cline { 2 - 4 } & Medium-term & One to five years long & Better than short-termed intervention \\
\cline { 2 - 4 } & Long-term & More than five years & Creates more persistent effect \\
\hline
\end{tabular}

Notes: We used water, sanitation, and hygiene (WASH)-related prevention health education and promotional interventions, (e.g., systematic reviews, empirical evidence) to explore the major features and concerns.

\section{Figures}

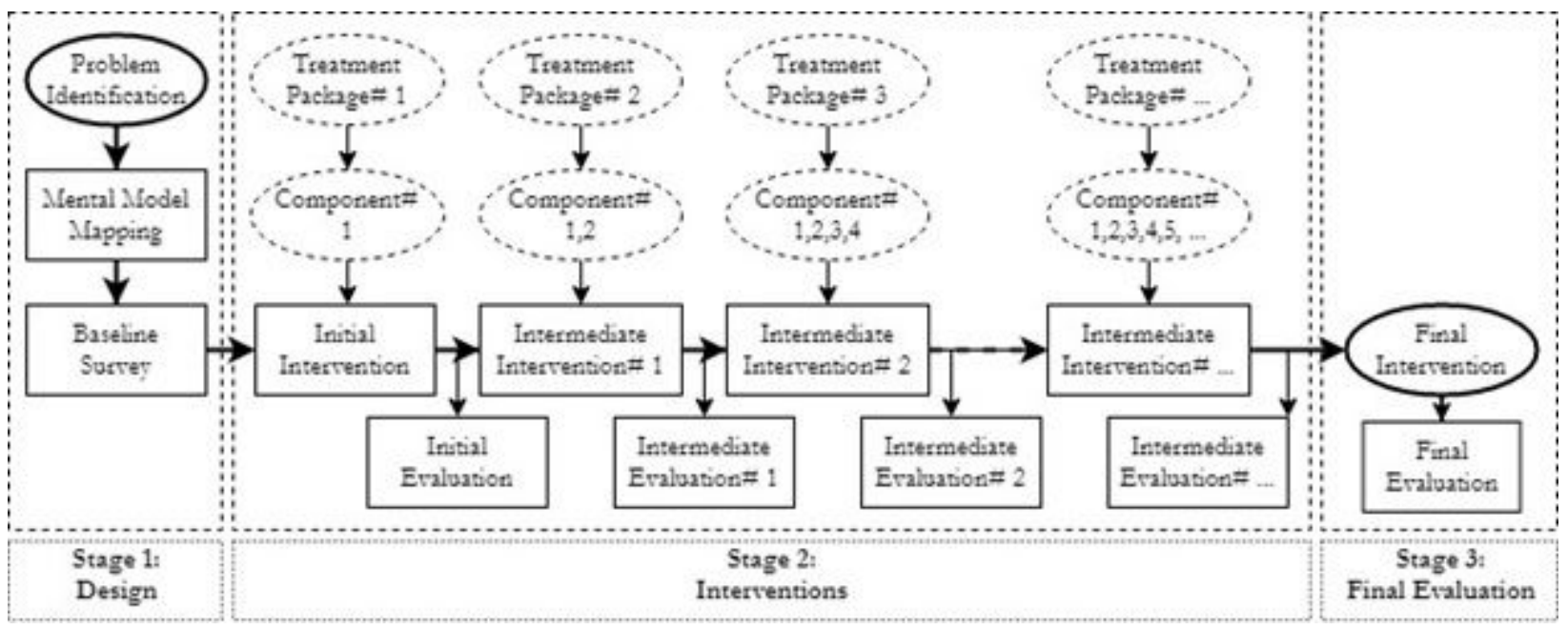

\section{Figure 1}

Conceptual Framework for Mental Model-based Repeated Multifaceted (MRM) Intervention Design. 